OPEN ACCESS

Edited by: Doris Wagner, University of Pennsylvania, USA

Reviewed by:

Gorou Horiguchi,

Rikkyo University, Japan Elizabeth Barker

University of Regina, Canada

*Correspondence:

Amy Litt

amy.litt@ucr.edu

Specialty section:

This article was submitted to Plant Evolution and Development, a section of the journal

Frontiers in Plant Science

Received: 30 September 2015

Accepted: 17 November 2015

Published: 02 December 2015

Citation:

McCarthy EW, Mohamed A and Litt A

(2015) Functional Divergence of APETALA1 and FRUITFULL is due

to Changes in both Regulation

and Coding Sequence.

Front. Plant Sci. 6:1076.

doi: 10.3389/fpls.2015.01076

\section{Functional Divergence of APETALA1 and FRUITFULL is due to Changes in both Regulation and Coding Sequence}

\author{
Elizabeth W. McCarthy' ${ }^{1}$, Abeer Mohamed ${ }^{2}$ and Amy Litt ${ }^{1 *}$ \\ ${ }^{1}$ Department of Botany and Plant Sciences, University of California, Riverside, Riverside, CA, USA, ${ }^{2}$ Department of \\ Agricultural Botany, Faculty of Agriculture (Saba Basha), Alexandria University, Alexandria, Egypt
}

Gene duplications are prevalent in plants, and functional divergence subsequent to duplication may be linked with the occurrence of novel phenotypes in plant evolution. Here, we examine the functional divergence of Arabidopsis thaliana APETALA1 (AP1) and FRUITFULL (FUL), which arose via a duplication correlated with the origin of the core eudicots. Both $A P 1$ and FUL play a role in floral meristem identity, but $A P 1$ is required for the formation of sepals and petals whereas FUL is involved in cauline leaf and fruit development. AP1 and FUL are expressed in mutually exclusive domains but also differ in sequence, with unique conserved motifs in the $\mathrm{C}$-terminal domains of the proteins that suggest functional differentiation. To determine whether the functional divergence of $A P 1$ and FUL is due to changes in regulation or changes in coding sequence, we performed promoter swap experiments, in which FUL was expressed in the AP1 domain in the ap1 mutant and vice versa. Our results show that FUL can partially substitute for $A P 1$, and $A P 1$ can partially substitute for FUL; thus, the functional divergence between $A P 1$ and FUL is due to changes in both regulation and coding sequence. We also mutated AP1 and FUL conserved motifs to determine if they are required for protein function and tested the ability of these mutated proteins to interact in yeast with known partners. We found that these motifs appear to play at best a minor role in protein function and dimerization capability, despite being strongly conserved. Our results suggest that the functional differentiation of these two paralogous key transcriptional regulators involves both differences in regulation and in sequence; however, sequence changes in the form of unique conserved motifs do not explain the differences observed.

\footnotetext{
Keywords: gene duplication, functional divergence, APETALA1, FRUITFULL, MADS box genes, conserved protein
} motifs

\section{INTRODUCTION}

Gene duplications are prevalent in angiosperms, occurring via either whole genome or tandem duplications. Duplications can increase robustness of developmental processes through redundancy (Wagner, 2008) or lead to the evolution of novel or partitioned functions between duplicates through the process of neo- or subfunctionalization (Ohno, 1970; Force et al., 1999; Lynch and Force, 2000). The increase in morphological complexity observed during the evolution 
of plants and animals is thought to be linked to functional divergence of gene duplicates (Ohno, 1970; Freeling and Thomas, 2006). MADS box transcription factors play key roles in the gene networks directing the floral transition and floral and fruit development and through duplication have diversified dramatically in plants, particularly in seed plants (Theissen et al., 2000; Kaufmann et al., 2005). The retention of gene duplicates drove this increase in gene number, and the diversity in function that occurred subsequent to duplication contributed to the development of the complex reproductive structures that are unique to these plant lineages (Theissen et al., 2000; Kaufmann et al., 2005).

One MADS-box gene subfamily that arose via duplications is the angiosperm-specific AP1/FUL lineage, the members of which play key roles in several important developmental processes including flower and fruit development. Multiple duplications have occurred in this lineage, including a key event that coincided with the origin of the core eudicots; this duplication produced the euAP1 (including Arabidopsis AP1) and euFUL (including Arabidopsis FUL) clades (Litt and Irish, 2003). This duplication is likely part of the whole genome triplication that occurred before the diversification of the core eudicots, often referred to as the gamma event (Jiao et al., 2012). In Arabidopsis, AP1 is required for proper specification of floral meristem identity and for sepal and petal development; in strong ap1 mutants, petals are not formed and sepals are transformed into bract-like organs (Irish and Sussex, 1990; Bowman et al., 1993). Secondary flowers can grow from the axils of these first whorl organs, and reiterate the phenotype, so that tertiary and quaternary flowers can occur (Irish and Sussex, 1990), indicating partial retention of inflorescence identity. In Arabidopsis ful mutants, fruit development is disrupted. Cells in the valves of the silique fail to elongate and differentiate, resulting in a short fruit; seed development proceeds as normal, which leads to over-crowding and premature rupture of the fruit wall ( $\mathrm{Gu}$ et al., 1998). In addition, the cauline leaves of $f u l$ mutants are wider than those of wild type (WT; Gu et al., 1998). AP1 excludes FUL from the floral meristem (Mandel and Yanofsky, 1995a), and the single ful mutant shows no defects in flower development ( $\mathrm{Gu}$ et al., 1998). However, in ap1-1 mutants, FUL is ectopically expressed in the floral meristem and further loss of floral identity is observed when FUL function is lost as well, indicating that FUL also is capable of specifying floral meristem identity; however, it cannot fully compensate for loss of AP1 function (Ferrandiz et al., 2000). Thus, $A P 1$ and FUL are redundant for one function, floral meristem identity, but otherwise have diverged functionally, playing distinct roles in perianth identity and in cauline leaf and fruit development, respectively.

Although their sequences are similar, as is expected from paralogs, the AP1 and FUL proteins have differing conserved motifs in their C-terminal domains. All FUL proteins, as well as the related SEPALLATA (SEP) and AGAMOUS-LIKE 6 (AGL6) proteins, have a six hydrophobic amino acid motif (LPAWML), the FUL-like motif, near the C terminus (Litt and Irish, 2003; Zahn et al., 2005; Shan et al., 2007). The function of this motif is unknown. AP1 has lost this FUL-like motif, due to a single nucleotide frame shift in the $3^{\prime}$ end of the coding sequence (Litt and Irish, 2003; Vandenbussche et al., 2003). Instead AP1 proteins have a transcription activation domain (Cho et al., 1999) and terminate in a farnesylation (a type of prenylation) domain (Yalovsky et al., 2000; Litt and Irish, 2003). The farnesylation domain directs the addition of a lipid moiety to the $\mathrm{C}$ terminus of the protein, and AP1 proteins have been shown to be farnesylated in vivo (Yalovsky et al., 2000). Farnesylation is often implicated in targeting proteins to membranes, and can be a mechanism for regulating transcription factor activity (Resh, 2006). Alternatively, it has been suggested that this motif may mediate protein interactions (Yalovsky et al., 2000). Overexpression of a mutated version of the AP1 protein, in which farnesylation was abolished, in WT Arabidopsis did not completely recapitulate the $A P 1$ overexpression phenotype and additionally displayed novel phenotypes, suggesting a role for this protein modification in AP1 function (Yalovsky et al., 2000). However, overexpression of related proteins which lack the farnesylation motif can also induce a phenotype similar to that of AP1 overexpression (Berbel et al., 2001; Blázquez et al., 2001; Castillejo et al., 2005; Chen et al., 2008). Thus the importance of these motifs to protein function is not clear; however, the presence of these sequence differences in the C-terminal protein domains, as well as other sequence differences between the proteins, is one possible explanation for the differences in function of AP1 and FUL.

A second possible explanation is that $A P 1$ and $F U L$ are expressed in mutually exclusive domains in Arabidopsis. AP1 is first expressed throughout young stages 1 and 2 floral meristems, but by stage 3 expression is restricted to the periphery of the floral meristem, where first and second whorl organs will arise (Mandel et al., 1992). AP1 expression is maintained in sepal and petal primordia as flowers develop (Mandel et al., 1992). In contrast, FUL expression is first seen in the inflorescence meristem at the onset of reproductive development and is found in the inflorescence meristem, the stem, and cauline leaves as inflorescence development continues (Mandel and Yanofsky, 1995a). FUL is not expressed in the floral meristem until stage 3, at which time it is found in the central dome, where the fourth whorl organs will arise; in later floral development, FUL is expressed in the valves of the developing carpels (Mandel and Yanofsky, 1995a). Thus, there is no overlap in the WT expression patterns of $A P 1$ and $F U L$, at least partly because AP1 represses FUL expression (Mandel and Yanofsky, 1995a), presenting another possible explanation for the functional differences seen between AP1 and FUL.

Pre-duplication FUL-like genes show broad expression patterns, with transcript generally present in the shoot apical meristem, leaves, inflorescence and floral meristems, and in most if not all floral organs and fruits (e.g., Yu and Goh, 2000; Pelucchi et al., 2002; Murai et al., 2003; Tsaftaris et al., 2004; Kim et al., 2005; Li et al., 2005; Preston and Kellogg, 2006; Pabón-Mora et al., 2012, 2013; Acri-Nunes-Miranda and Mondragón-Palomino, 2014; Sun et al., 2014). Basal eudicots belong to the same clade as the core eudicots, but diverged prior to the duplication that created the euAP1 and euFUL clades. $F U L$-like genes in these species are involved in regulation of flowering time, inflorescence branching, and cauline leaf, sepal, 
petal, carpel, and fruit development, essentially encompassing all the functions of $e u A P 1$ and $e u F U L$ genes combined (Pabón-Mora et al., 2012; however, see Pabón-Mora et al., 2013). This suggests that subfunctionalization and partitioning of the ancestral FULlike functions among the euAP1 and euFUL genes occurred following the core eudicot duplication. These data from FULlike genes set the stage for examination of the post-duplication evolutionary patterns of $e u A P 1$ and $e u F U L$ gene lineages.

Here, we examine the basis of functional differentiation between AP1 and FUL in Arabidopsis thaliana to determine if observed differences are due to their mutually exclusive expression domains or differences in their protein sequences. We perform promoter swap experiments and also investigate the role of conserved motifs in protein function through site-directed mutagenesis. In addition, because MADS-domain proteins act in complexes, we evaluated the ability of the mutated proteins to bind with known AP1 and FUL MADS-domain protein interacting partners. Our results suggest that the functional divergence of $A P 1$ and $F U L$ is due to changes in both regulation and coding sequence, and that the conserved motifs of AP1 and FUL may not play major roles in protein function.

\section{MATERIALS AND METHODS}

\section{Plant Material and Growth Conditions}

The following mutant lines were obtained from The Arabidopsis Information Resource (TAIR): CS28, ap1-1 mutant in a Ler background; CS3759, ful-1 mutant in a Ler background. The ap11 mutation (hereafter referred to as ap1) is a strong allele in which there is a mutation in the splice acceptor site of the third intron, resulting in transcript in which the third intron is retained (Mandel et al., 1992). The ful-1 mutation (hereafter referred to as ful) was produced via transposon-mediated enhancer trap mutagenesis, and the insertion of a DsE element into the $5^{\prime}$ UTR of the FUL gene yields a null mutation (Gu et al., 1998). WT CS20 Ler seeds were kindly provided by Michael Purugganan (New York University, New York, NY, USA). These lines were grown under $16 \mathrm{~h}$ light and $8 \mathrm{~h}$ dark at $21^{\circ} \mathrm{C}$ and $60 \%$ humidity.

\section{Cloning of AP1 and FUL Genes}

To clone the AP1 and FUL promoters, genomic DNA was extracted from WT CS20 Ler plants using phenol:chloroform (see Supplemental Materials and Methods for details). The 1.7 kilobase (kb) AP1 promoter (Hempel et al., 1997; AlvarezBuylla et al., 2006) was amplified using primers GL373F and GL374R, cloned into pCR 2.1-TOPO (Invitrogen), and sequenced in both directions. For the FUL promoter, we used a $4.2 \mathrm{~kb}$ fragment upstream of the FUL coding sequence that includes all of the identified upstream regulatory sequence (Nguyen, 2008; Woods, 2010). The FUL promoter was amplified using primers $\mathrm{AN} 19 \mathrm{~F}$ and $\mathrm{AN} 20 \mathrm{R}$, cloned into $\mathrm{pCR}$ XL-TOPO, and sequenced in both directions. A second amplification was performed to add a SacI restriction site to the $5^{\prime}$ end of the FUL promoter (primers GL395F and AN20R); this product was also cloned into pCR XL-TOPO and sequenced in both directions. Promoter sequences used also contained the $5^{\prime}$ UTR of the
mRNA. Primer sequences are found in Supplementary Table S1, and PCR conditions are found in Supplemental Materials and Methods.

To clone the AP1 and FUL coding sequences, RNA was extracted from inflorescence tissue of WT CS20 Ler plants using the RNeasy Plant Mini Kit (Qiagen) and DNase treated using the TURBO DNase-free kit (Ambion), according to manufacturers' instructions. Two micrograms of RNA were reverse transcribed into cDNA using SuperScript III (Invitrogen) according to the manufacturer's protocol. The AP1 coding sequence with the $3^{\prime}$ UTR was amplified using primers GL1090F and GL1091R, cloned into pCR 2.1-TOPO, and sequenced in both directions. The FUL coding sequence with the $3^{\prime}$ UTR was amplified using primers GL314F and GL315R, cloned into pCR 4-TOPO, and sequenced in both directions. Coding sequences used began with the start codon and included the $3^{\prime}$ UTR.

\section{Cloning of Constructs}

Chimeric PCR was performed to link the promoters and coding sequences and, with site-directed mutagenesis, to produce mutated coding sequences. Chimeric PCR consists of three PCR reactions, the first of which (PCR1) amplifies the $5^{\prime}$ portion of the final product, and the second of which (PCR2) amplifies the $3^{\prime}$ portion; these two products contain complementary and overlapping sequence. The third PCR (PCR3) uses both of these products as template, and amplification links them together into a single product. To link promoters and coding sequences, the first PCR used a forward primer that anneals to the $5^{\prime}$ end of the promoter and a reverse primer that binds to the $3^{\prime}$ end of the promoter, but also includes sequence complementary to the beginning of the coding sequence (Supplementary Figure S1A). The second PCR used a forward primer that binds to the $5^{\prime}$ end of the coding sequence and that includes sequence complementary to the $3^{\prime}$ end of the promoter. The reverse primer binds to the $3^{\prime}$ end of the coding sequence (Supplementary Figure S1A). The third PCR used the products of the first two PCRs as mixed templates and primers that bind to the $5^{\prime}$ end of the promoter and the $3^{\prime}$ end of the coding sequence. Because of the design of the internal primers (the reverse primer from PCR1 and the forward primer from PCR2), the products from the first two PCRs were complementary across the promoter/coding sequence link and annealed together; extension and amplification resulted in a seamless link between promoter and coding sequence (Supplementary Figure S1A).

Site-directed mutagenesis and chimeric PCR were also used to create mutated coding sequences. In this case, the internal primers were complementary and annealed across the target motif (Supplementary Figure S1B). The primers themselves included base pair mismatches that introduced point mutations to create the mutated coding sequences required. The third PCR used primers that amplified the entire coding sequence.

\section{Promoter Swap Constructs}

In order to examine whether FUL can substitute for AP1 function when expressed in the AP1 domain and vice versa, we created the promoter swap constructs, $P A P 1: F U L$ and $p F U L: A P 1$ along with the positive control constructs, $P A P 1: A P 1$ and $P F U L: F U L$ 
(Figure 1). We performed chimeric PCRs in which PCR1 amplified the promoter, PCR2 amplified the coding sequence, and PCR3 linked them (see above for description of chimeric PCR). The $p A P 1: F U L$ construct was produced using primers AN21F and AN22R for PCR1, AN24F and GL315R for PCR2, and GL373F and GL315R for PCR3, whereas the $p A P 1: A P 1$ construct was created using primers AN21F and AN23R for PCR1, AN25F and GL1419R for PCR2, and AN21F and AN15R for PCR3. These full length promoter:coding sequence constructs (PCR3 products) were cloned into pCR TOPO-2.1, and sequenced in both directions.

In order to prevent the introduction of errors via unnecessary reamplification of the long, previously cloned FUL promoter, we used a combination of chimeric PCR and restriction digests in the creation of the $p F U L: A P 1$ and $p F U L: F U L$ constructs. Chimeric PCR was performed to link the $A P 1$ and FUL coding sequences with a $200 \mathrm{bp}$ fragment of the $3^{\prime}$ end of the FUL promoter, using, for pFUL:AP1, primers AN10F and AN12R for PCR1, AN14F and AN15R for PCR2, and AN10F and AN15R for PCR3, and for pFUL:FUL, primers AN10F and AN11R for PCR1, AN13F and GL315R for PCR2, and AN10F and GL315R for PCR3. The $200 \mathrm{bp}$ fragment of the promoter includes a naturally occurring AccI restriction site, and the reverse primer in PCR3 introduced a BamHI restriction site at the end of the $3^{\prime}$ UTRs. PCR3 products, which consisted of $200 \mathrm{bp}$ of the promoter and the coding sequence with the BamHI site, were cloned into pCR 2.1-TOPO and sequenced in both directions. Restriction digest with AccI [New England Biolabs (NEBs)] and BamHI (NEB) was performed on both the full-length FUL promoter clone in the pCR TOPO-XL vector and on PCR3 clones. Fragments of the appropriate length were excised from an agarose gel, purified using the QIAEX II Gel Extraction Kit (Qiagen), ligated using T4 DNA ligase (NEB), and sequenced in both directions.

\section{Mutated Protein Constructs}

Site-directed mutagenesis and chimeric PCR were used to create mutated coding sequences in order to examine the role of conserved motifs in protein function. To abolish the AP1 farnesylation motif, following Yalovsky et al. (2000), the

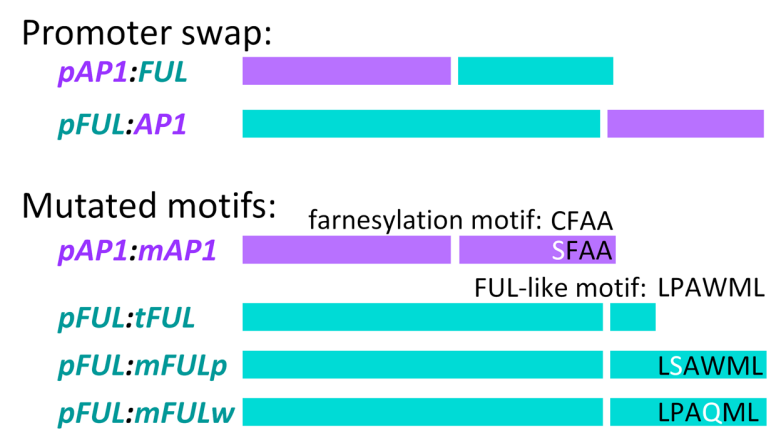

FIGURE 1 | Promoter swap and mutated motif constructs. In each construct, the left-hand of the two bars represents the promoter (color-coded, purple for AP1 and blue for FUL) and the right hand bar represents the coding sequence (similarly color coded) Descriptions of the constructs are in the text. receptor cysteine was replaced by serine to create the $m A P 1$ mutated coding sequence (Figure 1). Reactions used primers GL1090F and AN186R to amplify from the start codon to the farnesylation motif (PCR1), AN185F and AN15R to amplify from the farnesylation motif to the end of the $3^{\prime}$ UTR (PCR2), and GL1090F and AN15R to amplify the entire mutated coding sequence (PCR3).

To evaluate the function of the FUL-like motif, we constructed three mutated coding sequences (Figure 1). In all three cases, PCR1 amplified from the start codon to the FUL-like motif, PCR2 amplified from the FUL-like motif to the end of the $3^{\prime}$ UTR, and PCR3 amplified the entire mutated coding sequence. To produce the $t F U L$ mutated coding sequence, a stop codon was introduced just before the FUL-like motif, truncating the FUL protein and eliminating the motif; $t F U L$ was produced using primers GL314F and GL769R for PCR1, GL768F and GL315R for PCR2, and GL314F and GL315R for PCR3. To generate the mFULp mutated coding sequence, which resulted in a protein in which the nonpolar proline of the FUL-like motif (LPAWML) was replaced with a polar serine, we used primers GL10F and GL1238R for PCR1, GL1237F and GL315R for PCR2, and GL314F and GL315R for PCR3. To create the $m F U L w$ mutated coding sequence, which resulted in a protein in which the non-polar tryptophan of the FUL-like motif was replaced with a polar glutamine, we used primers GL10F and GL1236R for PCR1, GL1235F and GL315R for PCR2, and GL314F and GL315R for PCR3.

Chimeric PCR was used to link the AP1 and FUL promoters to the various mutated coding sequences. PCR1 amplified the promoter, PCR2 amplified the coding sequence, and PCR3 linked the promoter and coding sequence. The $p A P 1: m A P 1$ construct was created using primers AN21F and AN23R for PCR1, AN25F and GL1419R for PCR2, and AN21F and AN15R for PCR3. Products from PCR3 were cloned into PCR TOPO-2.1 and sequenced in both directions.

For the pFUL:tFUL, pFUL:mFULp, and pFUL:mFULw constructs, primers AN10F and AN11R were used for PCR1, AN13F and GL315R for PCR2, and AN10F and GL315R for PCR3. The PCR3 product for each mutated coding sequence was cloned into pCR TOPO-2.1 and sequenced in both directions. Restriction digests, gel excision and purification, ligation, and sequencing were performed as described above.

\section{Cloning into Binary Vectors}

All constructs were reamplified to introduce the sequence CACC at the $5^{\prime}$ end of the promoter for directional cloning. $p A P 1: A P 1$ and $p A P 1: m A P 1$ were amplified using primers AN305F and AN15R; $p A P 1: F U L$ was amplified using primers AN305F and AN306R; pFUL:FUL, pFUL:tFUL, pFUL:mFULp, and pFUL:mFULw were amplified using primers AN303F and GL315R; and pFUL:AP1 was amplified using primers AN304F and GL1419R. The resulting PCR products were cloned into pENTR/D-TOPO (Invitrogen) and sequenced. Constructs with the AP1 promoter were recombined into the Gateway $\mathrm{pK} 7 \mathrm{WG}$ binary vector, and those with the FUL promoter were recombined into the pH7WG binary vector (Department of Plant Systems Biology, Ghent University, Belgium) using LR Clonase II (Invitrogen). Empty pK7WG and pH7WG vectors were created 
by recombination with an empty $\mathrm{pENTR/D-TOPO}$ vector in order to remove the $c c d \mathrm{~B}$ screening gene. Each construct was transformed into Agrobacterium tumefaciens strain GV3101 (pMP90).

\section{Plant Transformation}

Floral dip transformation with Agrobacterium was performed following Clough and Bent (1998). Briefly, Agrobacterium cultures were resuspended in infiltration medium $(0.5 \mathrm{x}$ Murashige and Skoog salts with Gamborg's vitamins, 5\% sucrose, $0.05 \%$ MES, $0.044 \mu \mathrm{M}$ benzylaminopurine, $0.02 \%$ Silwet L-77) to $\mathrm{OD}_{600}=0.8$. Plants (ap1 mutants for constructs using the AP1 promoter and ful mutants for constructs using FUL promoter) were dipped into the Agrobacterium solution for $15 \mathrm{~min}$ and then pots were placed on their sides, covered to maintain humidity, and left in the dark overnight. Pots were placed upright, returned to the growth chamber ( $16 \mathrm{~h}$ light and $8 \mathrm{~h}$ dark, $21^{\circ} \mathrm{C}, 60 \%$ humidity), and left to grow until siliques matured. Seed was collected and screened for transformants.

\section{Transformant Screening}

$\mathrm{T}_{1}$ and $\mathrm{T}_{2}$ seed from all constructs was screened on MS plates (1x Murashige and Skoog with Gamborg's vitamins, 1\% sucrose, $0.05 \%$ MES, $0.8 \%$ agar) with the appropriate antibiotic: $50 \mu \mathrm{g} / \mathrm{mL}$ kanamycin for pK7WG constructs and $15 \mu \mathrm{g} / \mathrm{mL}$ hygromycin for $\mathrm{pH} 7 \mathrm{WG}$ constructs (hygromycin plates had no sucrose). Seedlings on plates were grown under $16 \mathrm{~h}$ light and $8 \mathrm{~h}$ dark at $21^{\circ} \mathrm{C}$ and $60 \%$ humidity, and seedlings that grew true leaves were deemed putative transformants and transferred to soil.

RNA was extracted from putative transformants from inflorescence tissue for ap 1 mutants and from inflorescence and silique tissue for ful mutants, and cDNA was synthesized as described above. The resulting cDNA was screened for presence of transgene insertion using PCR for kanamycin, using primers GL418F and GL419F, or hygromycin, using primers AN210F and AN211R, and for expression of the construct using the following strategies.

The ap 1-1 mutant has a point mutation that disrupts the splice acceptor site for the third intron, but transcript is still expressed (Mandel et al., 1992). Therefore, we designed screening primers (primers AN334F and AN215R) that amplify only the WT $A P 1$ transcript from our $p A P 1: A P 1$ and $p A P 1: m A P 1$ constructs, but not the endogenous transcript from the ap1 mutant (see Results for further information). Similarly, to distinguish between expression of FUL transcript from our $P A P 1$ :FUL construct and endogenous FUL mRNA in ap1 mutants, we designed a forward primer in the AP1 5' UTR and a reverse primer in the FUL coding sequence (primers AN299F and AN301R).

The ful-1 mutation is an insertion of a DsE transposable enhancer trap element in the $5^{\prime}$ UTR of the FUL gene, and no mRNA transcript is produced ( $\mathrm{Gu}$ et al., 1998). However, to be certain that we were only amplifying expression from our constructs, we designed a forward primer (AN379F) that includes sequence on either side of the DsE insertion site in order to screen for expression in the ful-1 mutant background. To screen for the expression of the pFUL:FUL, $p F U L: t F U L, p F U L: m F U L p$, and $p F U L: m F U L w$ constructs in putative transformants, we used primers AN379F and AN380R. To screen for the expression of the pFUL:AP1 construct, we used primers AN379F and AN381R. To distinguish between transcripts expressed from $p F U L: F U L$, pFUL:tFUL, pFUL:mFULp, and pFUL:mFULw constructs, we designed additional reverse primers across the engineered point mutations in the FUL-like motif: AN383R for $t F U L$, AN385R for mFULp, and AN386R for mFULw, all used with forward primer AN382F. We used actin as a control (primers AN221F and AN222R). Primer sequences are found in Supplementary Table S1, and PCR conditions are found in Supplemental Materials and Methods.

\section{Scoring Transformant Phenotypes}

Both $\mathrm{T}_{1}$ and $\mathrm{T}_{2}$ transformants were scored for phenotypes; results are reported for $\mathrm{T}_{2}$ plants. For experiments that tested the ability of a construct to complement the ap1 mutant ( $p A P 1: F U L a p 1$ and $p A P 1: m A P 1$ ap 1 lines), flowers 1-5 and 11-15 were scored for the number of flowers per pedicel. In addition, the number of petals per flower and the identity of first and second whorl organs were recorded for both primary and secondary flowers for flowers 1-5 and 11-15. For experiments that tested the ability of a construct to complement the ful mutant ( $p F U L: A P 1$ ful, $p F U L: t F U L$ ful, $p F U L: m F U L p f u l$, and $p F U L: m F U L w$ ful lines), cauline leaf length and width were scored for the primary inflorescence and flowers 1-15 were scored for silique length. ANOVAs and Tukey's Honest Significant Difference tests were performed in RStudio version 0.98.490, and Bonferroni corrections were applied to all statistical tests conducted.

\section{Yeast Two-hybrid Analysis}

Yeast two-hybrid analyses were performed to determine whether the mutated AP1 and FUL coding sequences created here could still bind with known MADS-domain AP1 and FUL interaction partners. Yeast two-hybrid vectors were obtained from TAIR (www.arabidopsis.com) for AGAMOUS-LIKE 24 (AGL24), AP1, APETALA3 (AP3), FUL, PISTILLATA (PI), SEP1, and SUPPRESSOR OF CONSTANS1 (SOC1) and created for mAP1, tFUL, mFULp, mFULw, AGAMOUS (AG), AGL6, SEP3, SEP4-II, and SHORT VEGETATIVE PHASE (SVP). We amplified full-length coding sequences using the following primer combinations: AG (primers AN237F and AN238R), AGL6 (primers AN235F and AN373R), SEP3 (primers AN233F and AN234R), SEP4-II (primers AN241F and AN242R), and SVP (primers AN239F and AN240R). We amplified the fulllength coding sequences for tFUL, mFULp, and mFULw using primers AN246F and AN247R. AP1 has been reported to have autoactivation capability (Pelaz et al., 2001), so we used chimeric PCR to create additional vectors (designated AP1PGA and mAP1-PGA) for both WT AP1 and mAP1 in which the proline- and glutamine-rich regions and the activation domain were removed from the C-terminal region of the protein (Supplementary Figure S2A). We used this approach instead of protein truncation because we were evaluating whether the farnesylation motif at the $\mathrm{C}$ terminus of the protein is necessary for protein-protein interactions. We performed chimeric PCR in which PCR1 amplified from the start codon until just before the proline-rich domain, PCR2 amplified from just after 
the activation domain to the stop codon, and PCR3 linked these two regions, creating a coding sequence from which the proline- and glutamine-rich regions and the activation domain were removed. For AP1-PGA, we used the following primers: AN243F and AN248R (PCR1), AN249F and AN244R (PCR2), and AN243F and AN244R (PCR3). For mAP1-PGA, we used the following primers: AN243F and AN248R (PCR1), AN249F and AN245R (PCR2), and AN243F and AN245R (PCR3). These vectors were no longer capable of autoactivation (Supplementary Figure S2B). Full-length coding sequences were cloned into pENTR/D-TOPO, sequenced in both directions, and recombined into both pDEST-AD and pDEST-DB vectors, kindly provided by David Hall (Dana-Farber Cancer Institute, Boston, MA, USA), using LR Clonase II (Invitrogen). Interactions were tested by co-transforming pairs of vectors into yeast strain AH109. AP1, mAP1, AP1-PGA, mAP1-PGA, FUL, tFUL, mFULp, and mFULw were each tested against a panel of interactors: AG, AGL6, AGL24, AP3, PI, SEP1, SEP3, SEP4-II, SOC1, and SVP in both pDEST-AD and pDEST-DB vectors. Empty pDEST$\mathrm{AD}$ and pDEST-DB vectors were added to the panel as negative controls. Co-transformed yeast was plated onto selective plates (synthetic drop-out; -HWL with 0, 2.5, 5, 10, 20, and $30 \mathrm{mM} 3 \mathrm{AT}$, -AWL, and -AHWL) and growth was monitored after 3 and 6 days. The interaction patterns of the mutated proteins were compared to those of their WT counterparts to determine whether the mutation disrupted normal proteinprotein interactions.

\section{RESULTS}

\section{The ap1-1 Mutant Produces Multiple Transcripts}

In developing a strategy to screen for expression of the $p A P 1: A P 1$ and $p A P 1: m A P 1$ constructs in the ap1-1 mutant background, we discovered that the ap1 transcript pool is variable in this mutant. The ap1-1 mutant has a point mutation in the splice acceptor site of the third intron and is predicted to produce a longer fragment that includes the third intron. We originally designed screening primers across the third and fourth exon boundary, but amplification yielded a 'wild type' sized fragment as well as the expected longer one. Sequencing established that transcripts of three different lengths are expressed. These included a long transcript (1118 bp) in which the third intron is retained, a short transcript (925 bp) in which the third intron, fourth exon, and fourth intron are spliced out, and a 'wild type' length transcript (1023 bp) in which the third intron has been spliced out along with one extra base pair. This 'wild type' length transcript occurs because the point mutation creates a second 'AG' splice acceptor site one base pair downstream of the original site; splicing of the transcript at this site yields a frame shift mutation. To avoid confusion when using PCR amplification to screen for construct expression, we designed new primers (AN334F/AN215R, see Supplementary Table S1 for sequences) for screening transformants that only amplify WT AP1 transcript, utilizing the single nucleotide difference between WT and the 'wild type' length ap1-1 mutant transcripts.

\section{The Basis of Functional Divergence between AP1 and FUL}

$A P 1$ and FUL have different functions, are expressed in mutually exclusive domains and are divergent in sequence. To determine if the functional divergence between $A P 1$ and FUL is due to changes in regulation, we created promoter swap constructs in which the FUL coding sequence is driven by the AP1 promoter ( $p A P 1: F U L)$ and vice versa ( $p F U L: A P 1)$. If differences in expression underlie the observed functional differences, we would expect our promoter swap constructs to completely complement the corresponding mutant phenotype. If differences in sequence contribute to the functional divergence, we would not expect these constructs to rescue the mutants. We therefore introduced the $p A P 1$ :FUL construct into the strong ap1-1 mutant and the $p F U L: A P 1$ construct into the strong ful-1 mutant. Plants transformed with the promoter swap constructs were compared to WT and the corresponding mutant as well as positive ( $p A P 1: A P 1$ ap1 or $p F U L: F U L$ ful ) and negative (empty vector) controls.

\section{FUL Can Partially Rescue the ap1 Mutant When Expressed in the AP1 Domain}

Wild type flowers have one flower per pedicel with four sepals and four petals (Figures $2 \mathbf{A}, \mathbf{D}-\mathbf{H}$ ). In contrast, ap1 mutant flowers have multiple flowers per pedicel, and these flowers have predominantly bract-like organs in the first whorl (Figures 2C,E-G). However, we observed some carpelloid bracts (in which stigmatic papillae and/or ovules are formed), a few unfused carpels (with stigmatic papillae at the distal end and ovules along the margins), and some filamentous organs in the first whorl in ap1 mutants (Figures 2F,G). Approximately half of both first and second whorl organs are missing (Figures 2F-H); when second whorl organs are present, they are mainly stamen-like or filamentous structures, but a few bract-like organs are observed as well (Figure $\mathbf{2 H}$ ). The stamen-like organs are either stamens (with anthers), petaloid stamens (in which petal tissue is fused to the anther), or carpelloid stamens (in which carpelloid structures are either fused to the anther or replace the anther on the filament; Figure $\mathbf{2 H}$ ). Our observations of second whorl organs differ from published descriptions of the ap1-1 mutant in that we see a greater percentage of organs in this whorl (53\% as opposed to $6 \%$ in Bowman et al., 1993 and none in Irish and Sussex, 1990); however, the types of organs we observe are consistent with what Bowman et al. (1993) recorded for other ap1 alleles. The $p A P 1: A P 1$ construct (the positive control) rescues the phenotype to WT; these lines have one flower per pedicel, with petals and predominantly normal sepals, although some sepals have the Y-shaped trichomes characteristic of leaves (Supplementary Figure S3). Empty vector ap1 lines resemble ap1 mutants (Supplementary Figure S3).

The promoter swap $p A P 1: F U L$ ap1 lines, in which the FUL coding sequence was expressed in the AP1 domain in an ap1 mutant background, have mainly bract-like organs in the first whorl; however, we also observed carpelloid bracts, filamentous structures, and a few unfused carpels, similar to $a p 1$ 

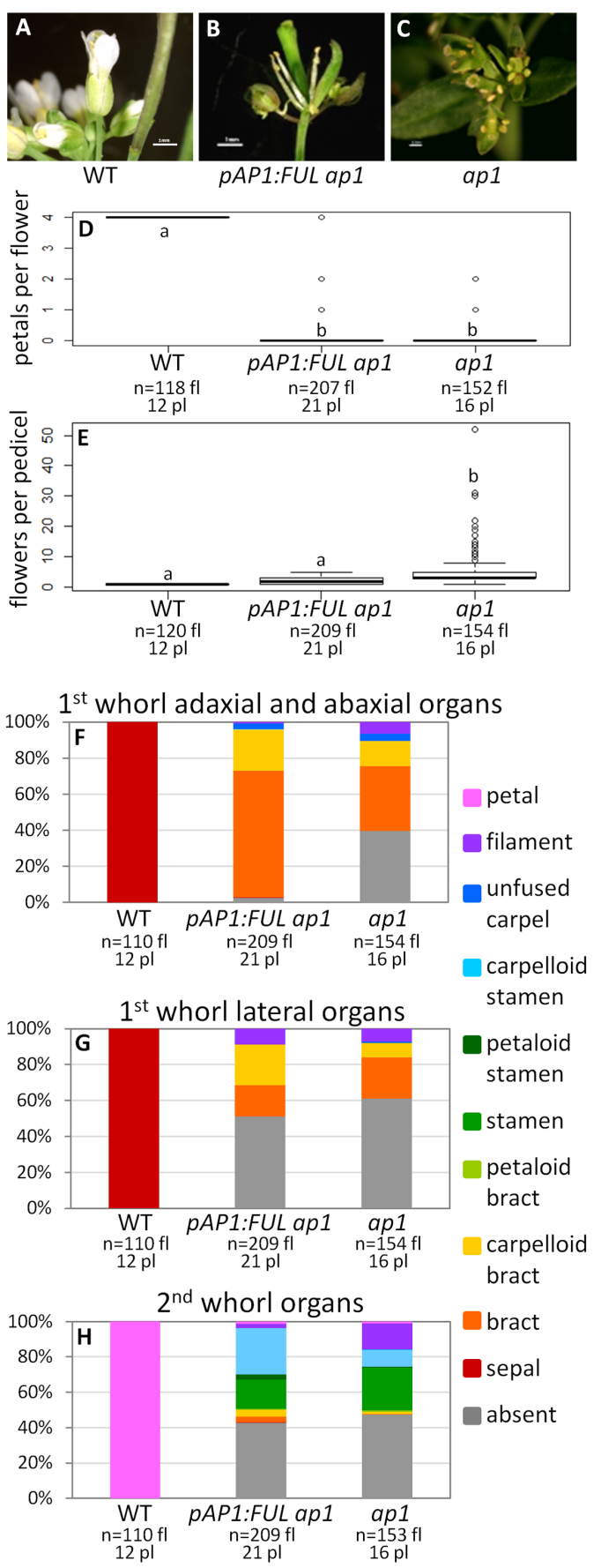

FIGURE 2 | FUL can partially substitute for $\boldsymbol{A P 1}$. Flowers from wild type (WT) (A), pAP1:FUL ap1 (B), and ap1 mutants (C). Box plots showing the number of petals per flower (D) and number of flowers per pedicel (E) in WT, pAP1:FUL ap1 lines, and ap1 mutants. Lowercase letters in box plots denote significance; boxes with the same letter are not significantly different from each other according to ANOVAs and Tukey's Honest Significant Difference tests and following Bonferroni corrections. Cumulative bar graphs describing the identity of first whorl adaxial and abaxial organs (F), first whorl lateral organs (G), and second whorl organs $(\mathbf{H})$ in WT, pAP1:FUL ap1 lines, and ap1 mutants. Sample size is noted below each line. The top number is the number of flowers scored, and the bottom number is the number of plants from which these flowers came. mutants (Figures 2B,F,G). These lines have no petals (ANOVA: $F=17530, \mathrm{df}=4, p<2 \times 10^{-16}$ ), and approximately half of the second whorl organs are missing, but when present, they are mainly stamen-like and filamentous structures, similar to the ap1 mutant (Figures 2B,D-H). However, 98\% of adaxial and abaxial first whorl organs are present in the $p A P 1$ :FUL ap1 lines, compared to only $61 \%$ in ap1 mutants (Figures 2B,F,G). The identity of the first and second whorl organs of the secondary flowers (which arise from the axils of first whorl organs) is also the same as in ap1 mutants (ANOVA: $F=137.1$, df $=2$, $p<2 \times 10^{-16}$; Supplementary Figure S4). However, in contrast to ap1 mutants, $p A P 1$ :FUL ap1 lines show a reduced number of flowers per pedicel with an average of 2.04 compared to 5.34 in ap1 mutants and 1.0 in WT (ANOVA: $F=109.2, \mathrm{df}=4$, $p<2 \times 10^{-16}$; Figure 2E). Although $p A P 1$ :FUL ap1 lines are not significantly different from WT in number of flowers per pedicel after Bonferroni correction, they do show some secondary flowers whereas WT plants never do. Although no petals are produced in $p A P 1: F U L$ ap1 lines, the reduced number of flowers per pedicel and the increase in the number of first whorl organs in these lines show that FUL can at least partially substitute for AP1 not only in floral meristem identity, as expected, but also in some elements of floral structure.

\section{AP1 Can Partially Rescue the ful Mutant When Expressed in the FUL Domain}

Wild type plants produce siliques with an average length of $11.47 \mathrm{~mm}$ and their cauline leaves have a width:length ratio of 0.48 (Figures $\mathbf{3 A}, \mathbf{D}, \mathbf{H}, \mathbf{I}$ ). In contrast, ful mutants have siliques with an average length of $3.73 \mathrm{~mm}$ and a cauline leaf width:length ratio of 0.63 (Figures $3 \mathbf{C , F}, \mathbf{H}, \mathbf{I}$ ). The positive control pFUL:FUL construct rescues the ful mutant phenotype, with an average silique length of $9.63 \mathrm{~mm}$. This is similar to, but slightly shorter than, siliques in WT, but significantly longer than the ful mutant siliques (ANOVA: $F=3995$, df $=4, p<2 \times 10^{-16}$; Supplementary Figure S5). The cauline leaf width:length ratio in pFUL:FUL ful lines is 0.45 and is not significantly different from WT (ANOVA: $F=106.7$, df $=4, p<2 \times 10^{-16}$; Supplementary Figure S5). Empty vector $f u l$ lines resemble $f u l$ mutants (Supplementary Figure S5).

The pFUL:AP1 ful lines, in which the AP1 coding sequence is expressed in the FUL domain in a ful mutant background, have lumpy, sometimes curved siliques that are significantly longer $(5.30 \mathrm{~mm})$ than $f u l$ mutants, but significantly shorter than WT (ANOVA: $F=3995, \mathrm{df}=4, p<2 \times 10^{-16}$; Figures 3B,H). The pFUL:AP1 ful lines have curled cauline leaves with an average width:length ratio of 0.55 ; this is significantly larger than the WT average of 0.48 , but significantly smaller than the average of 0.63 seen in ful mutants (ANOVA: $F=106.7, \mathrm{df}=4$, $p<2 \times 10^{-16}$; Figure 3I). The $p F U L: A P 1$ ful lines have terminal flowers (Figure 3G), which are not present in WT plants; these likely result from $A P 1$ expression in the inflorescence meristem under the control of the FUL promoter. Longer siliques and relatively narrower cauline leaves in the $p F U L: A P 1 \mathrm{ful}$ lines show that $A P 1$ can partially substitute for FUL when expressed in the FUL domain. 


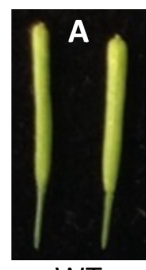

WT

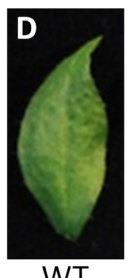

WT
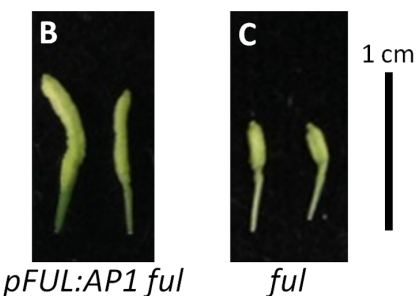

pFUL:AP1 ful

ful
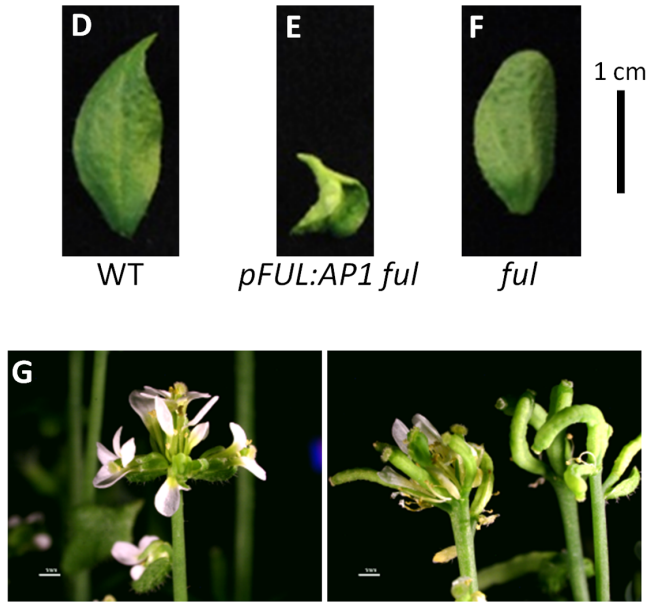

pFUL:AP1 ful
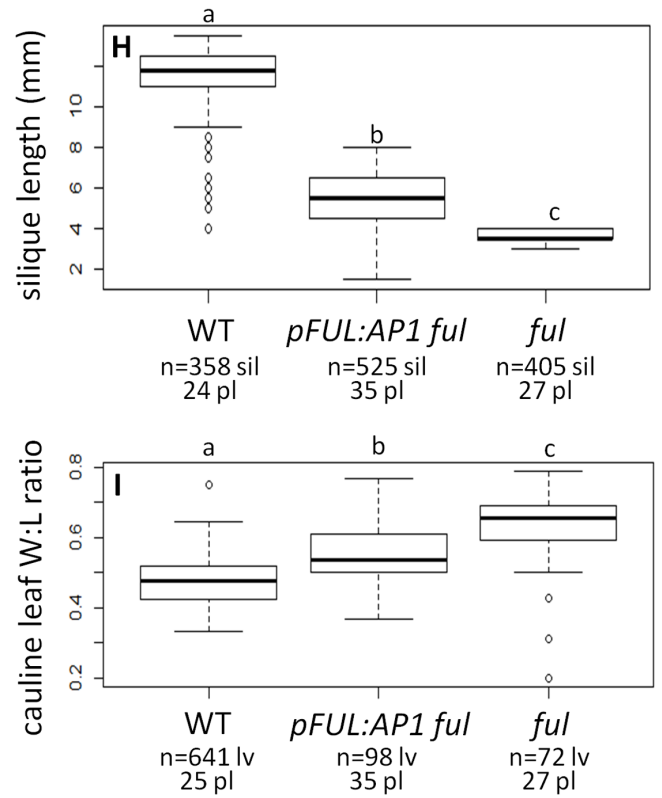

FIGURE 3 | AP1 can partially substitute for FUL. Siliques of WT (A), pFUL:AP1 ful (B), and ful mutants (C). Cauline leaves of WT (D), pFUL:AP1 ful (E), and ful mutants (F). Terminal flower phenotype of pFUL:AP1 ful lines (G). Box plots showing silique length in millimeters $\mathbf{( H )}$ and cauline leaf width:length $(\mathrm{W}: \mathrm{L})$ ratio (I) for WT, pFUL:AP1 ful lines, and ful mutants. Lowercase letters in box plots denote significance; boxes with the same letter are not significantly different from each other according to ANOVAs and Tukey's Honest Significant Difference tests and following Bonferroni corrections. Sample size is noted below each line. The top number is the number of siliques or cauline leaves scored, and the bottom number is the number of plants from which they came.

\section{The Function of Conserved Motifs} The Farnesylation Motif is not Required for AP1

\section{Function}

To determine if the AP1 farnesylation motif is required for protein function, we generated a construct in which the receptor cysteine was mutated to a serine ( $P A P 1$ :mAP1; Figure 1), which prevents the attachment of the farnesyl molecule. The pAP1:mAP1 ap1 lines resemble WT; they have only one flower per pedicel (ANOVA: $F=138.5$, df $=4, p<2 \times 10^{-16}$; Figures $4 \mathrm{~A}, \mathrm{~B}, \mathrm{D}-\mathrm{H}$ ), and those flowers have petals (ANOVA: $F=18495, \mathrm{df}=4, p<2 \times 10^{-16}$ ) and sepals (sometimes with Y-shaped trichomes). The fact that the mutated AP1 protein can complement the ap1 mutant shows that farnesylation of the AP1 protein is not necessary for proper protein function.

\section{FUL Protein Function is Largely not Dependent on the FUL-like Motif}

We examined whether the FUL-like motif is necessary for FUL protein function by creating three mutated coding sequences: $t F U L$, which creates a protein that is truncated just before the FUL-like motif; mFULp, in which the proline in the FUL-like motif is replaced with a serine; and mFULw, in which the tryptophan is replaced with a glutamine. The tryptophan in the fourth position of the motif is strictly conserved in all proteins from the AP1/FUL, SEP, and AGL6 lineages, and the proline in the second position is conserved across angiosperm sequences (Litt and Irish, 2003). In both mFULp and mFULw, a non-polar amino acid was replaced with a polar amino acid, disrupting the hydrophobic motif.

Average silique length was similar in all three sets of plants transformed with mutated FUL constructs: pFUL:tFUL ful (8.10 mm), pFUL:mFULp ful $(8.09 \mathrm{~mm})$, and pFUL:mFULw ful $(8.52 \mathrm{~mm})$. These lengths were significantly longer than ful mutants $(3.73 \mathrm{~mm})$, but significantly shorter than WT (11.47 mm; ANOVA: $F=1895, \mathrm{df}=6, p<2 \times 10^{-16}$; Figures $\mathbf{5 A}-\mathbf{E}, \mathbf{K}$ ), suggesting that this conserved amino acid motif may have a minor role in silique elongation. The cauline leaf width:length ratio in all three mutated FUL ful lines was not significantly different than WT (0.48); however, the ratio in pFUL:mFULp ful lines (0.45) is significantly smaller than in pFUL:tFUL ful (0.51) and pFUL:mFULw ful (0.57) lines (ANOVA: $F=108.8, \mathrm{df}=6, p<2 \times 10^{-16}$; Figures $\left.5 \mathrm{~F}-\mathbf{J}, \mathbf{L}\right)$. This observation, that a protein lacking the proline is more successful at complementing the phenotype than one lacking the tryptophan or lacking the motif entirely, suggests that the proline plays a less significant role than the tryptophan in protein function. Nonetheless, none of the ratios were significantly different from WT, suggesting that this motif is not required for proper cauline leaf development. These results, which show that the mutated protein constructs complement the ful cauline leaf phenotype and partially complement the silique defect, suggest that the FUL-like motif plays only a minor role in FUL protein function.

\section{Protein-protein Interactions}

MADS-domain proteins are thought to act in multimeric complexes (Egea-Cortines et al., 1999; Honma and Goto, 2001; 

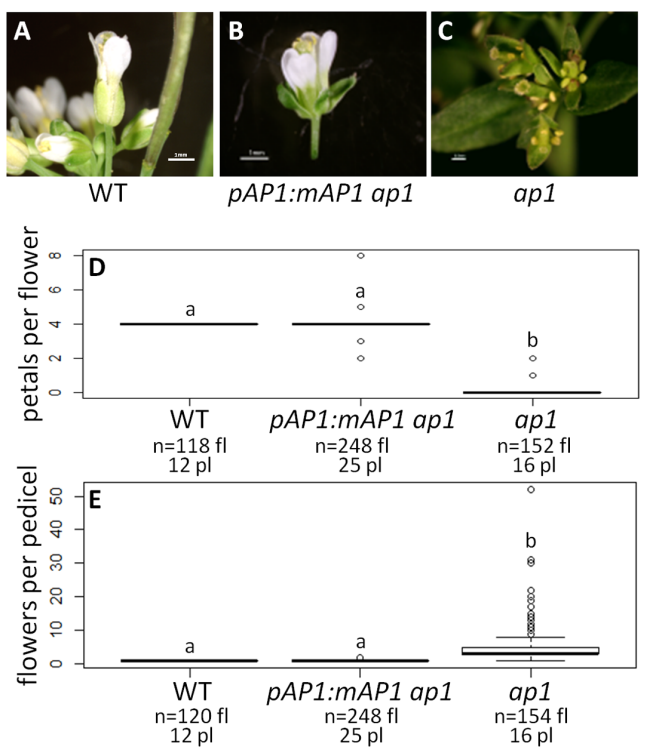

$1^{\text {st }}$ whorl adaxial and abaxial organs
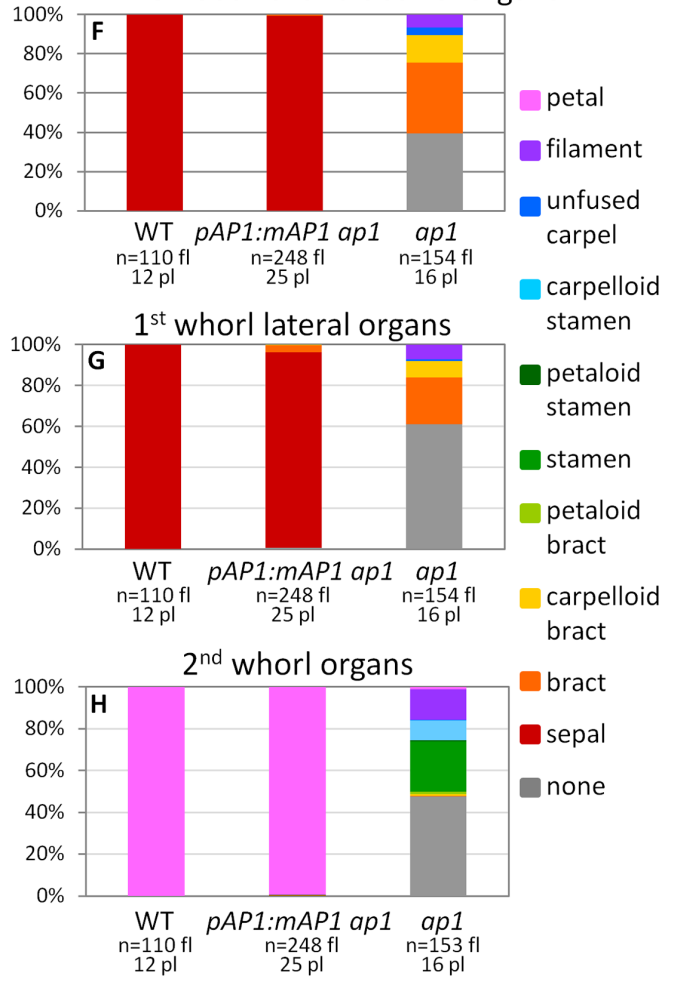

FIGURE 4 | AP1 farnesylation motif is not necessary for protein function. Flowers from WT (A), pAP1:mAP1 ap1 (B), and ap1 mutants (C). Box plots showing the number of petals per flower (D) and the number of flowers per pedicel (E) for WT, pAP1:mAP1 ap1, and ap1 mutants. Lowercase letters in box plots denote significance; boxes with the same letter are not significantly different from each other according to ANOVAs and Tukey's Honest Significant Difference tests and following Bonferroni corrections. Cumulative bar graphs describing the identity of first whorl adaxial and abaxial organs $(\mathbf{F})$, first whorl lateral organs $(\mathbf{G})$, and second whorl organs $\mathbf{( H )}$ in WT, pAP1:mAP1 ap1 lines, and ap1 mutants. Sample size is noted below each line. The top number is the number of flowers scored, and the bottom number is the number of plants from which these flowers came.
Theissen, 2001; Theissen and Saedler, 2001; Melzer and Theissen, 2009; Melzer et al., 2009). Yalovsky et al. (2000) suggested that farnesylation, which adds a hydrophobic tail to the $\mathrm{C}$ terminus of the AP1 protein, might mediate such interactions. Furthermore, the conserved FUL-like motif consists of hydrophobic amino acids, suggesting a possible role in protein-protein interactions. We therefore conducted yeast two-hybrid experiments to determine whether mutations in these motifs disrupt known protein-protein interactions. AP1 is capable of autoactivation, so yeast two-hybrid constructs were made in which the prolineand glutamine-rich regions and activation domain were removed from the $\mathrm{C}$ terminus of the protein for both AP1 and mAP1 (Supplementary Figure S2).

In our experiments, AP1 and FUL proteins interacted with AGL6, AGL24, SEP1, SEP3, and SVP, but not AG, AP3, PI, SEP4-II, or SOC1 (Figure 6). The MAP1 protein interactions were identical (Figure 6), indicating that, at least in yeast, the farnesylation motif is not required for dimerization with the MADS-domain proteins we investigated. FUL proteins with mutated motifs also have largely the same interaction partners as WT when grown on lower stringency plates (-HLW with $20 \mathrm{mM}$ 3AT). An exception is that mFULp, in which the proline of the FUL-like motif is replaced by a serine, interacts with AG (Figure 6A), whereas WT protein does not, under our conditions. However, this interaction is no longer seen on higher stringency plates (-HLW with $30 \mathrm{mM}$ 3AT; Figure 6B). The interactions of tFUL (truncated) and mFULw (tryptophan replaced with glutamine) with AGL6 and SVP are also weaker or absent on the higher stringency plates (Figure 6B), suggesting that the FUL-like motif may play at least some role in protein-protein interactions.

\section{DISCUSSION}

\section{Both Changes in Regulation and Coding Sequence Underlie Functional Differentiation of $A P 1$ and FUL}

Our results show that the FUL coding sequence can partially complement the ap1 mutant when expressed in the AP1 domain and that the AP1 coding sequence can partially rescue the ful mutant when expressed in the FUL domain (Figures 2 and 3 ). These results suggest that the divergence in function between these two genes is the result of changes in coding sequence as well as changes in regulation. Our pFUL:AP1 ful lines show partial rescue of the silique length and cauline leaf shape defects of the ful mutant (Figures 3A-F,H,I), showing that the AP1 protein has some ability, albeit limited, to substitute for FUL. Our $p A P 1: F U L$ ap1 lines had fewer flowers per pedicel, showing that FUL can moderate the inflorescence meristem defects of $a p 1$ (Figure 2E). This is consistent with the fact that FUL, as well as AP1, is capable of promoting floral meristem identity (Ferrandiz et al., 2000). The pAP1:FUL ap1 lines also had an increase in first whorl organ number. However, no petals are formed in these lines (Figure 2D), showing that FUL does not have all of the functional capabilities of AP1. Previous experiments expressing a FUL-like gene from the grass Lolium temulentum in the Arabidopsis ap1 

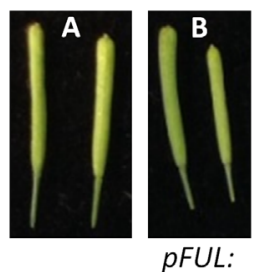

WT

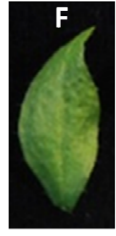
ful
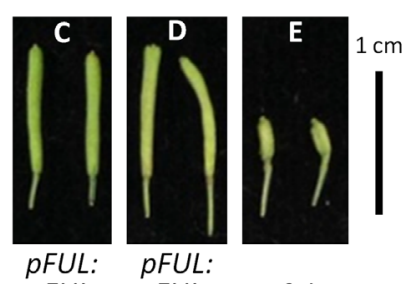

pFUL:

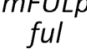

ful
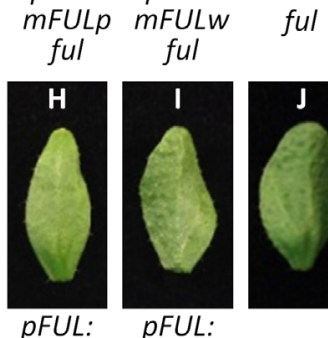

pFUL:

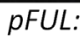

WT

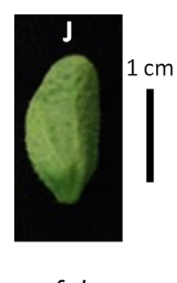

ful ful $^{\text {ful }}$ ful
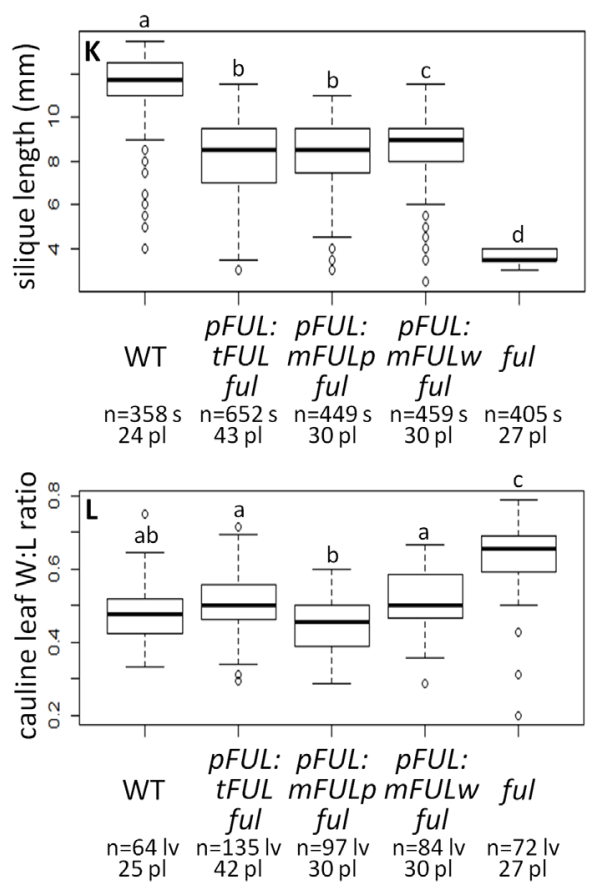

FIGURE 5 | FUL-like motif plays a minor role in silique elongation. Siliques from WT (A), pFUL:tFUL ful (B), pFUL:mFULp ful (C), pFUL:mFULw ful (D), and ful mutants (E). Photographs of cauline leaves from WT (F), pFUL:tFUL ful (G), pFUL:mFULp ful (H), pFUL:mFULw ful (I), and ful mutants (J). Box plots showing silique length in millimeters (K) and cauline leaf width:length (W:L) ratio (L) for WT, pFUL:tFUL ful, pFUL:mFULp ful, pFUL:mFULW ful, and ful mutants. Lowercase letters in box plots denote significance; boxes with the same letter are not significantly different from each other according to ANOVAs and Tukey's Honest Significant Difference tests and following Bonferroni corrections. Sample size is noted below each line. The top number is the number of siliques or cauline leaves scored, and the bottom number is the number of plants from which they came.

mutant under the control of the AP1 promoter similarly showed that this gene could not rescue the petal defect but was able to partially complement the defects in flower number per pedicel and first whorl organ number (Gocal et al., 2001). The fact that expression of either $A P 1$ or FUL in the domain of the other produces only partial mutant complementation indicates that the proteins are not functionally equivalent, and that differences in sequence do have functional consequences.

\section{Conserved AP1 and FUL Amino Acid Motifs are not Necessary for Protein Function \\ AP1 farnesylation motif}

Our mutated AP1 ap1 lines, in which the receptor cysteine of the farnesylation motif was replaced with serine thus preventing farnesylation of the AP1 protein, show complete complementation of the mutant phenotype (Figure 4). The only exception is that sepals sometimes have Y-shaped trichomes, which is also seen in our $p A P 1: A P 1$ ap 1 positive control lines. These results suggest that this post-translational modification of the AP1 protein, which has been demonstrated to occur in planta (Yalovsky et al., 2000), is not necessary for proper AP1 function. Yalovsky et al. (2000) generated the same mutated AP1 protein, in which the receptor cysteine was replaced with serine, and expressed it in WT Arabidopsis plants under the cauliflower mosaic virus (CaMV) 35S promoter (Benfey et al., 1989). Although their mutated AP1 lines flowered early, similar to what is seen when WT AP1 is overexpressed, these lines failed to show the terminal flower phenotype that is typical of AP1 overexpression (Mandel and Yanofsky, 1995b). Instead they displayed novel phenotypes, including increased density of trichomes on rosette leaves and sepals and sometimes lack of chlorophyll in leaves and inflorescences; this led them to conclude that farnesylation played an important role in AP1 function (Yalovsky et al., 2000). Because overexpression phenotypes may not reflect the function of a protein during normal plant development, we instead expressed the mutated $A P 1$ sequence under the control of the WT AP1 promoter in the ap1 mutant to see if it could replace the WT protein. Our results suggest that it can, and that addition of a farnesyl molecule is not required for normal AP1 function. Nevertheless, Yalovsky et al. (2000) did show that the protein is farnesylated in Arabidopsis, thus the purpose of this modification remains unknown.

Evidence from studies with $A P 1 / F U L$ genes from other species also suggests that this post-translational modification is not required for AP1 function. The AP1 ortholog in pea, PEAM4, lacks a farnesylation motif, but can restore petal production when constitutively expressed in Arabidopsis ap1-1 mutants (Berbel et al., 2001). The average number of petals per flower in these lines is less than that seen in WT or 35S:AP1 lines (Berbel et al., 2001), but this may be due to additional sequence changes that arose since the divergence of Arabidopsis and pea. Similarly, overexpression of a euFUL gene from tobacco and FUL-like genes from Lilium and rice, which all lack the farnesylation motif, produced petals; although, in fewer numbers than in WT plants (Jang et al., 2002; Chen et al., 2008).

Arabidopsis has another AP1 paralog, CAULIFLOWER (CAL), which arose from a more recent duplication than $A P 1$ and FUL. $C A L$ possesses a farnesylation motif, but kinetic analyses suggest that it is unlikely to be farnesylated in planta (Yalovsky et al., 

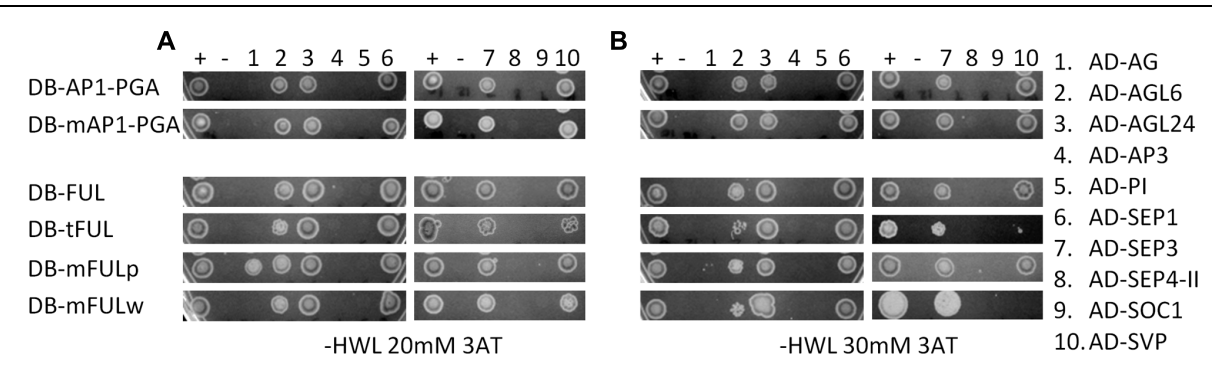

FIGURE 6 | The FUL-like, but not the farnesylation, motif may play a role in mediating protein interactions. Interactions in yeast on -HWL 20 mM $3 A T$ (A) and -HWL 30 mM 3AT (B) plates after 6 days of growth. Mutated proteins were compared with WT AP1 and FUL proteins to determine whether altering or abolishing the conserved motifs disrupted protein-protein interactions. Numbers above the images refer to proteins listed in the key on the right hand side. Constructs fused to the binding domain are labeled "DB"; constructs fused to the activation domain are labeled "AD." Proteins and controls are described in the text. + and - indicate positive and negative (empty vector) control. AP1-PGA, mAP1-PGA = AP1 protein and AP1 protein with mutated farnesylation motif lacking the proline-rich $(P)$, glutamine-rich $(G)$, and activation (A) domains to abolish autoactivation (see Supplementary Figure S2A for diagram).

2000). When expressed under the $A P 1$ promoter, $C A L$ cannot rescue the ap1-1 mutant phenotype; however, chimeric proteins, in which the M-, I-, and K-domains of AP1 are fused to the C-domain of CAL, nearly completely rescue ap1-1 (AlvarezBuylla et al., 2006). This suggests that the inability of CAL to substitute for AP1 is due to sequence in the M-, I-, and $\mathrm{K}$-domains, and not the C-terminal domain that contains the farnesylation motif.

\section{FUL-like Motif}

The ful mutant phenotype is nearly completely rescued by all three of our mutated FUL constructs (Figure 5), in which either the entire motif was absent or the most highly conserved residues were substituted. This suggests that the FUL protein can function nearly normally without the FUL-like motif; however, silique length in all three mutated FUL ful lines is significantly shorter than WT (Figures 5A-E,K), indicating that the FUL-like motif plays at least some role in silique elongation.

The FUL-like motif is highly conserved not only in all euFUL proteins, but also in the FUL-like proteins found in plant lineages outside the core eudicots (which predate the euAP1/euFUL duplication), and in the closely related SEP and AGL6 gene clades. These latter genes are also implicated in flowering and floral development, and arose via duplication from the same lineage as AP1/FUL (Litt and Irish, 2003). Only the AGL6 lineage predates angiosperms, being found in gymnosperms as well; $A P 1 / F U L$ and SEP genes are restricted to flowering plants and are required for flowering (Theissen et al., 2000; Litt and Irish, 2003). The tryptophan in the fourth position of the six amino acid motif appears to be strictly conserved across not only FUL and FUL-like proteins, but also the SEP and AGL6 lineages (Litt and Irish, 2003). This suggests an important function for this residue; however, our results suggest only a minor role in silique elongation. The proline in the second position is conserved in angiosperm euFUL and FUL-like proteins (Litt and Irish, 2003), and similarly, only seems to play a minor role in silique elongation. Our results suggest that the mutated proline transcript may be better able to complement the cauline leaf width:length ratio defect of the mutant; nonetheless, none of these lines were significantly different from WT for this trait (Figures 5F-J,L). The fact that SEP proteins have this same motif, and that several SEP proteins are co-expressed with FUL (de Folter et al., 2005), suggests that SEP proteins may be able to substitute to some extent, although certainly not completely, for FUL, thereby masking the significance of the loss or alteration of the FUL-like motif in FUL.

Our promoter swap experiments show that sequence differences between AP1 and FUL have functional consequences; however, our results suggest that the relevant differences are not those of the highly conserved amino acid motifs present in the C-terminal domain. It goes against accepted wisdom to suggest that highly conserved motifs do not have functional significance. However, studies with a chimeric protein, in which the MADS and I domains of AP1 were fused to the K and C domains of AGAMOUS (AG), show that in fact that the specific sequence of the entire $\mathrm{C}$ terminus may not be required. This chimeric protein can provide nearly complete complementation of the ap1-1 mutant phenotype when driven by the AP1 promoter (Krizek et al., 1999), yet AG has entirely different conserved amino acid motifs in its C-terminal domain (Kramer et al., 2004). Studies with truncated APETALA3 (AP3) and PISTILLATA (PI) proteins that lack the conserved C-terminal motifs characteristic of those lineages (Piwarzyk et al., 2007) confirm that the conspicuous motifs of MADS-domain proteins are less significant than assumed. This raises the question of why they are so highly conserved, particularly as the rest of the C-terminal domain tends to be highly variable, even among closely related species.

\section{Altering or Abolishing Conserved Motifs Affects some Protein Interactions in Yeast}

Our yeast-two hybrid experiments reveal that abolishing the AP1 farnesylation motif does not alter protein-protein interactions (Figure 6). This indicates that the farnesylation motif is not necessary for dimerization with the MADS-domain proteins we tested; however, it is still possible that this motif is involved in interaction with other proteins or plays a role in the formation of multimeric complexes. In addition, it has not been shown that AP1 is farnesylated in yeast; although yeast has the machinery to 
perform this post-translational modification (Omer and Gibbs, 1994). Nevertheless, our transgenic experiments, which indicate that this motif is not required for protein function, support the idea that it is not required to mediate functionally relevant protein interactions.

In contrast, tFUL (in which the FUL protein terminates before the FUL-like motif) and mFULw (in which tryptophan is replaced with glutamine) have weaker interactions with AGL6 and SVP than are seen with FUL (Figure 6B). This indicates that the FUL-like motif may play a role in some protein-protein interactions, consistent with its hydrophobic nature. FUL-SVP heterodimers have been implicated in the regulation of meristem identity in the vegetative-reproductive transition (Balanzà et al., 2014). The specific role of FUL-AGL6 interactions has not been documented; however, both genes are co-expressed in floral development and are implicated in the regulation of flowering (Ohmori et al., 2009; Rijpkema et al., 2009; Thompson et al., 2009; Koo et al., 2010; Yoo et al., 2011). These interactions may be at least partly dependent on the FUL-like motif. We did not observe defects in flowering time in our transgenic experiments with mutated FUL proteins, suggesting that weak interactions may be sufficient to produce the appropriate developmental outcomes; alternatively, the program that promotes flowering contains significant redundancy which may compensate for any loss of interaction.

Our yeast-two hybrid results differ from those previously published: in contrast to our findings, de Folter et al. (2005) found that FUL did not interact with SVP, but did interact with AG, and that both FUL and AP1 interacted with SEP4II and SOC1. However, van Dijk et al. (2010) observed FULSVP interaction, similar to what we found, and Balanzà et al. (2014) confirmed this interaction in planta. Although this type of study allows comparison with previous published protein interaction studies, yeast-two hybrid experiments are known to produce both false positives and negatives, and to be sensitive to experimental conditions (Legrain and Selig, 2000); therefore, it is not surprising that results may vary. Also, the relevance of observed interactions to in planta processes must be verified.

Our yeast-two hybrid experiments were limited to MADSdomain proteins, but AP1 and FUL interact with proteins of other families as well, and it is possible that these interactions may be influenced by the C-terminal motifs. Nevertheless, the data from plants transformed with our mutated protein constructs suggest that any effects that are present do not produce significant phenotypic defects. This may be due to redundancy, particularly in the case of FUL, or it may simply be that these motifs are not as critical for proper function as assumed.

\section{Duplicate Genes Promote both Diversity and Redundancy in Developmental Networks}

Functional divergence of gene duplicates can result in maintenance of both copies in the genome and lead to new gene functions that can produce novel phenotypes and increased organismal complexity (Ohno, 1970; Freeling and Thomas, 2006). Functional divergence can be based on changes in expression, changes in sequence, or both. Our experiments indicate that in the case of the Arabidopsis paralogs AP1 and $F U L$, the third option has occurred; changes in both regulation and coding sequence have driven functional divergence. More recent paralogs, $A P 1$ and $C A L$, share a similar expression pattern, and $C A L$ is completely redundant with $A P 1$ function; although, the reverse is not true. Loss of $C A L$ function enhances the loss of floral meristem identity (Kempin et al., 1995), but CAL is not able to complement the floral organ defects of the ap1 mutant when driven by the AP1 promoter (Alvarez-Buylla et al., 2006). In fact, $C A L$ may be gradually losing its function; it has a higher rate of non-synonymous substitutions than AP1 (Liljegren et al., 1999) and fewer MADS-domain protein interaction partners than AP1 (de Folter et al., 2005). Amino acid differences at two positions between $A P 1$ and $C A L$ have been shown to account for a large portion of the interaction differences observed between the two proteins, and swapping these residues yielded gain or loss of some protein interaction partners in a yeast system (van Dijk et al., 2010). FUL shares one of these amino acid residues with AP1, which may explain why FUL is able to partially rescue the ap1 mutant when expressed in the AP1 domain (Figure 2), whereas $C A L$ cannot (Alvarez-Buylla et al., 2006).

Intriguingly, all three of these genes that are related by duplication retain the function of promoting floral meristem identity, although to different extents and in different contexts. In WT development, FUL does not play this role because it is excluded from the floral meristem by AP1 (Mandel and Yanofsky, 1995a), providing an example of how changes in regulation can result in functional divergence between duplicates. $A P 1$ and $C A L$ are expressed in similar domains, but $A P 1$ has functional capabilities that $C A L$ does not have (Kempin et al., 1995; Ferrandiz et al., 2000); thus sequence differences must be the differentiating factor. Nevertheless, all three genes are capable of contributing to floral meristem identity in a redundant fashion. This evidence supports the hypothesis that duplicate genes not only diversify and create novel complexity within developmental systems, but also that they strengthen already existing pathways to ensure robustness in important developmental processes, such as the transition to flowering.

\section{AUTHOR CONTRIBUTIONS}

AL developed the project. EM performed most of the experiments. AM cloned the AP1 promoter and FUL coding sequence, created the yeast-two hybrid constructs, and performed yeast-two hybrid experiments. EM and AL wrote the manuscript.

\section{ACKNOWLEDGMENT}

We thank Maya Strahl, Leah Reilly, and high school interns Christian Carrasco, Cary Chapman, Nina Naghshineh, and Caitlin Piccorillo-Stosser for technical assistance. 


\section{FUNDING}

This work was supported by NSF award IOS-0923748 and The New York Botanical Garden, New York, NY, USA.

\section{REFERENCES}

Acri-Nunes-Miranda, R., and Mondragón-Palomino, M. (2014). Expression of paralogous SEP-, FUL-, AG- and STK-like MADS-box genes in wild-type and peloric Phalaenopsis flowers. Front. Plant Sci. 5:76. doi: 10.3389/fpls.2014.00076

Alvarez-Buylla, E. R., García-Ponce, B., and Garay-Arroyo, A. (2006). Unique and redundant functional domains of APETALA1 and CAULIFLOWER, two recently duplicated Arabidopsis thaliana floral MADS-box genes. J. Exp. Bot. 57, 3099-3107. doi: 10.1093/jxb/erl081

Balanzà, V., Martínez-Fernández, I., and Ferrándiz, C. (2014). Sequential action of FRUITFULL as a modulator of the activity of the floral regulators SVP and SOC1. J. Exp. Bot. 65:1193. doi: 10.1093/jxb/ert482

Benfey, P. N., Ren, L., and Chua, N.-H. (1989). The CaMV 35 S enhancer contains at least two domains which can confer different developmental and tissue-specific expression patterns. EMBO J. 8, 2195-2202.

Berbel, A., Navarro, C., Ferrándiz, C., Cañas, L. A., Madueño, F., and Beltrán, J.-P. (2001). Analysis of PEAM4, the pea AP1 functional homologue, supports a model for AP1-like genes controlling both floral meristem and floral organ identity in different plant species. Plant J. 25, 441-451. doi: 10.1046/j.1365313x.2001.00974.x

Blázquez, M., Koornneef, M., and Putterill, J. (2001). Flowering on time: genes that regulate the floral transition: workshop on the molecular basis of flowering time control. EMBO Rep. 2, 1078-1082. doi: 10.1093/embo-reports/kve254

Bowman, J. L., Alvarez, J., Weigel, D., Meyerowitz, E. M., and Smyth, D. R. (1993). Control of flower development in Arabidopsis thaliana by APETALA1 and interacting genes. Development 119, 721-743.

Castillejo, C., Romera-Branchat, M., and Pelaz, S. (2005). A new role of the Arabidopsis SEPALLATA3 gene revealed by its constitutive expression. Plant $\mathrm{J}$. 43, 586-596. doi: 10.1111/j.1365-313X.2005.02476.x

Chen, M.-K., Lin, I.-C., and Yang, C.-H. (2008). Functional analysis of three lily (Lilium longiflorum) APETALA1-like MADS box genes in regulating floral transition and formation. Plant Cell Physiol. 49, 704-717. doi: $10.1093 / \mathrm{pcp} / \mathrm{pcn} 046$

Cho, S., Jang, S., Chae, S., Chung, K. M., Moon, Y.-H., An, G., et al. (1999). Analysis of the C-terminal region of Arabidopsis thaliana APETALA1 as a transcription activation domain. Plant Mol. Biol. 40, 419-429. doi: 10.1023/A:1006273127067

Clough, S. J., and Bent, A. F. (1998). Floral dip: a simplified method for Agrobacterium-mediated transformation of Arabidopsis thaliana. Plant J. 16, 735-743. doi: 10.1046/j.1365-313x.1998.00343.x

de Folter, S., Immink, R. G. H., Kieffer, M., Pařenicová, L., Henz, S. R., Weigel, D., et al. (2005). Comprehensive interaction map of the Arabidopsis MADS box transcription factors. Plant Cell 17, 1424-1433. doi: 10.1105/tpc.105.031831

Egea-Cortines, M., Saedler, H., and Sommer, H. (1999). Ternary complex formation between the MADS-box proteins SQUAMOSA, DEFICIENS and GLOBOSA is involved in the control of floral architecture in Antirrhinum majus. EMBO J. 18, 5370-5379. doi: 10.1093/emboj/18.19.5370

Ferrandiz, C., Gu, Q., Martienssen, R., and Yanofsky, M. F. (2000). Redundant regulation of meristem identity and plant architecture by FRUITFULL, APETALA1 and CAULIFLOWER. Development 127, 725-734.

Force, A., Lynch, M., Pickett, F. B., Amores, A., Yan, Y. L., and Postlethwait, J. (1999). Preservation of duplicate genes by complementary, degenerative mutations. Genetics 151, 1531-1545.

Freeling, M., and Thomas, B. C. (2006). Gene-balanced duplications, like tetraploidy, provide predictable drive to increase morphological complexity. Genome Res. 16, 805-814. doi: 10.1101/gr.3681406

Gocal, G. F. W., King, R. W., Blundell, C. A., Schwartz, O. M., Andersen, C. H., and Weigel, D. (2001). Evolution of floral meristem identity genes. Analysis of Lolium temulentum genes related to APETALA1 and LEAFY of Arabidopsis. Plant Physiol. 125, 1788-1801. doi: 10.1104/pp.125. 4.1788

\section{SUPPLEMENTARY MATERIAL}

The Supplementary Material for this article can be found online at: http://journal.frontiersin.org/article/10.3389/fpls.2015.01076

Gu, Q., Ferrandiz, C., Yanofsky, M. F., and Martienssen, R. (1998). The FRUITFULL MADS-box gene mediates cell differentiation during Arabidopsis fruit development. Development 125, 1509-1517.

Hempel, F. D., Weigel, D., Mandel, M. A., Ditta, G., Zambryski, P. C., Feldman, L. J., et al. (1997). Floral determination and expression of floral regulatory genes in Arabidopsis. Development 124, 3845-3853.

Honma, T., and Goto, K. (2001). Complexes of MADS-box proteins are sufficient to convert leaves into floral organs. Nature 409, 525-529. doi: 10.1038/35054083

Irish, V. F., and Sussex, I. M. (1990). Function of the apetala-1 gene during Arabidopsis floral development. Plant Cell 2, 741-753. doi: 10.1105/tpc.2.8.741

Jang, S., An, K., Lee, S., and An, G. (2002). Characterization of tobacco MADSbox genes involved in floral initiation. Plant Cell Physiol. 43, 230-238. doi: $10.1093 / \mathrm{pcp} / \mathrm{pcf} 015$

Jiao, Y., Leebens-Mack, J. H., Ayyampalayam, S., Bowers, J. E., McKain, M. R., McNeal, J., et al. (2012). A genome triplication associated with early diversification of the core eudictos. Genome Biol. 13:R3. doi: 10.1186/gb-201213-1-r3

Kaufmann, K., Melzer, R., and Theißen, G. (2005). MIKC-type MADS-domain proteins: structural modularity, protein interactions and network evolution in land plants. Gene 347, 183-198. doi: 10.1016/j.gene.2004.12.014

Kempin, S. A., Savidge, B., and Yanofsky, M. F. (1995). Molecular basis of the cauliflower phenotype in Arabidopsis. Science 267, 522-525. doi: 10.1126/science.7824951

Kim, S., Koh, J., Yoo, M.-J., Kong, H., Hu, Y., Ma, H., et al. (2005). Expression of floral MADS-box genes in basal angiosperms: implications for the evolution of floral regulators. Plant J. 43, 724-744. doi: 10.1111/j.1365-313X.2005.02487.x

Koo, S. C., Bracko, O., Park, M. S., Schwab, R., Chun, H. J., Park, K. M., et al. (2010). Control of lateral organ development and flowering time by the Arabidopsis thaliana MADS-box gene AGAMOUS-LIKE6. Plant J. 62, 807-816. doi: 10.1111/j.1365-313X.20 10.04192.x

Kramer, E. M., Jaramillo, M. A., and Di Stilio, V. S. (2004). Patterns of gene duplication and functional evolution during the diversification of the AGAMOUS subfamily of MADS box genes in angiosperms. Genetics 166, 1011-1023. doi: 10.1534/genetics.166.2.1011

Krizek, B. A., Riechmann, J. L., and Meyerowitz, E. M. (1999). Use of the APETALA1 promoter to assay the in vivo function of chimeric MADS box genes. Sex. Plant Reprod. 12, 14-26. doi: 10.1007/s004970050167

Legrain, P., and Selig, L. (2000). Genome-wide protein interaction maps using two-hybrid systems. FEBS Lett. 480, 32-36. doi: 10.1016/S0014-5793(00) 01774-9

Li, G.-S., Meng, Z., Kong, H.-Z., Chen, Z.-D., Theissen, G., and Lu, A.-M. (2005). Characterization of candidate class A, B and E floral homeotic genes from the perianthless basal angiosperm Chloranthus spicatus (Chloranthaceae). Dev. Genes Evol. 215, 437-449. doi: 10.1007/s00427-005-0002-2

Liljegren, S. J., Gustafson-Brown, C., Pinyopich, A., Ditta, G. S., and Yanofsky, M. F. (1999). Interactions among APETALA1, LEAFY, and TERMINAL FLOWER1 specify meristem fate. Plant Cell 11, 1007-1018. doi: 10.1105/tpc.11.6.1007

Litt, A., and Irish, V. F. (2003). Duplication and diversification in the APETALA1/FRUITFULL floral homeotic gene lineage: implications for the evolution of floral development. Genetics 165, 821-833.

Lynch, M., and Force, A. (2000). The probability of duplicate gene preservation by subfunctionalization. Genetics 154, 459-473.

Mandel, M. A., Gustafson-Brown, C., Savidge, B., and Yanofsky, M. F. (1992). Molecular characterization of the Arabidopsis floral homeotic gene APETALA1. Nature 360, 273-277. doi: 10.1038/360273a0

Mandel, M. A., and Yanofsky, M. F. (1995a). The Arabidopsis AGL8 MADS box gene is expressed in inflorescence meristems and is negatively regulated by APETALA1. Plant Cell 7, 1763-1771. doi: 10.1105/tpc.7.11.1763

Mandel, M. A., and Yanofsky, M. F. (1995b). A gene triggering flower formation in Arabidopsis. Nature 377, 522-524. doi: 10.1038/377522a0 
Melzer, R., and Theissen, G. (2009). Reconstitution of floral quartets in vitro involving class B and class E floral homeotic proteins. Nucleic Acids Res. 37, 2723-2736. doi: 10.1093/nar/gkp129

Melzer, R., Verelst, W., and Theissen, G. (2009). The class E floral homeotic protein SEPALLATA3 is sufficient to loop DNA in floral quartet-like complexes in vitro. Nucleic Acids Res. 37, 144-157. doi: 10.1093/nar/gkn900

Murai, K., Miyamae, M., Kato, H., Takumi, S., and Ogihara, Y. (2003). WAP1, a wheat APETALA1 homolog, plays a central role in the phase transition from vegetative to reproductive growth. Plant Cell Physiol. 44, 1255-1265. doi: $10.1093 / \mathrm{pcp} / \mathrm{pcg} 171$

Nguyen, A. L. (2008). Transcriptional Regulation of FRUITFULL: A MADS-box Gene Involved in Arabidopsis Fruit Development. Master thesis, University of California, San Diego, CA.

Ohmori, S., Kimizu, M., Sugita, M., Miyao, A., Hirochika, H., Uchida, E., et al. (2009). MOSAIC FLORAL ORGANS1, an AGL6-like MADS box gene, regulates floral organ identity and meristem fate in rice. Plant Cell 21, 3008-3025. doi: 10.1105/tpc.109.068742

Ohno, S. (1970). Evolution by Gene Duplication. Heidelberg: Springer-Verlag.

Omer, C. A., and Gibbs, J. B. (1994). Protein prenylation in eukaryotic microorganisms: genetics, biology and biochemistry. Mol. Microbiol. 11, 219-225. doi: 10.1111/j.1365-2958.1994.tb00302.x

Pabón-Mora, N., Ambrose, B., and Litt, A. (2012). Poppy APETALA1/FRUITFULL orthologs control flowering time, branching, perianth identity and fruit development. Plant Physiol. 158, 1685-1704. doi: 10.1104/pp.111.192104

Pabón-Mora, N., Sharma, B., Holappa, L. D., Kramer, E. M., and Litt, A. (2013). The Aquilegia FRUITFULL-like genes play key roles in leaf morphogenesis and inflorescence development. Plant J. 74, 197-212. doi: 10.1111/tpj.12113

Pelaz, S., Gustafson-Brown, C., Kohalmi, S. E., Crosby, W. L., and Yanofsky, M. F. (2001). APETALA1 and SEPALLATA3 interact to promote flower development. Plant J. 26, 385-394. doi: 10.1046/j.1365-313X.2001.2641042.x

Pelucchi, N., Fornara, F., Favalli, C., Masiero, S., Lago, C., Pè, E., et al. (2002). Comparative analysis of rice MADS-box genes expressed during flower development. Sex. Plant Reprod. 15, 113-122. doi: 10.1007/s00497-002-0151-7

Piwarzyk, E., Yang, Y., and Jack, T. (2007). Conserved C-terminal motifs of the Arabidopsis proteins APETALA3 and PISTILLATA are dispensable for floral organ identity function. Plant Physiol. 145, 1495-1505. doi: 10.1104/pp.107.105346

Preston, J. C., and Kellogg, E. A. (2006). Reconstructing the evolutionary history of paralogous APETALA1/FRUITFULL-like genes in grasses (Poaceae). Genetics 174, 421-437. doi: 10.1534/genetics.106.057125

Resh, M. D. (2006). Trafficking and signaling by fatty-acylated and prenylated proteins. Nat. Chem. Biol. 2, 584-590. doi: 10.1038/nchembio834

Rijpkema, A. S., Zethof, J., Gerats, T., and Vandenbussche, M. (2009). The petunia AGL6 gene has a SEPALLATA-like function in floral patterning. Plant J. 60, 1-9. doi: 10.1111/j.1365-313X.2009.03917.x

Shan, H., Zhang, N., Liu, C., Xu, G., Zhang, J., Chen, Z., et al. (2007). Patterns of gene duplication and functional diversification during the evolution of the AP1/SQUA subfamily of plant MADS-box genes. Mol. Phylogenet. Evol. 44, 26-41. doi: 10.1016/j.ympev.2007.02.016

Sun, W., Huang, W., Li, Z., Song, C., Liu, D., Liu, Y., et al. (2014). Functional and evolutionary analysis of the AP1/SEP/AGL6 superclade of MADS-box genes in the basal eudicot Epimedium sagittatum. Ann. Bot. 113, 653-668. doi: $10.1093 / \mathrm{aob} / \mathrm{mct} 301$
Theissen, G. (2001). Development of floral organ identity: stories from the MADS house. Curr. Opin. Plant Biol. 4, 75-85. doi: 10.1016/S1369-5266(00) 00139-4

Theissen, G., Becker, A., Di Rosa, A., Kanno, A., Kim, J., Münster, T., et al. (2000). "A short history of MADS-box genes in plants," in Plant Molecular Evolution, eds J. Doyle and B. Gaut (Amsterdam: Springer), 115-149.

Theissen, G., and Saedler, H. (2001). Plant biology - floral quartets. Nature 409, 469-471. doi: 10.1038/35054172

Thompson, B. E., Bartling, L., Whipple, C., Hall, D. H., Sakai, H., Schmidt, R., et al. (2009). Bearded-ear encodes a MADS box transcription factor critical for maize floral development. Plant Cell 21, 2578-2590. doi: 10.1105/tpc.109.067751

Tsaftaris, A. S., Pasentsis, K., Iliopoulos, I., and Polidoros, A. N. (2004). Isolation of three homologous AP1-like MADS-box genes in crocus (Crocus sativus L.) and characterization of their expression. Plant Sci. 166, 1235-1243. doi: 10.1016/j.plantsci.2003.12.037

Vandenbussche, M., Theissen, G., Van de Peer, Y., and Gerats, T. (2003). Structural diversification and neofuctionalization during floral MADS-box gene evolution by C-terminal frameshift mutations. Nucleic Acids Res. 31, 4401-4409. doi: 10.1093/nar/gkg642

van Dijk, A. D. J., Morabito, G., Fiers, M., van Ham, R. C. H. J., Angenent, G. C., and Immink, R. G. H. (2010). Sequence motifs in MADS transcription factors responsible for specificity and diversification of protein-protein interaction. PLoS Comput. Biol. 6:e1001017. doi: 10.1371/journal.pcbi.1001017

Wagner, A. (2008). Gene duplications, robustness and evolutionary innovations. BioEssays 30, 367-373. doi: 10.1002/bies.20728

Woods, J. C. (2010). Analysis of FRUITFULL Promoter Motifs and Their Influence on Valve Expression During Fruit Development. Master thesis, University of California, San Diego, CA.

Yalovsky, S., Rodríguez-Concepción, M., Bracha, K., Toledo-Ortiz, G., and Gruissem, W. (2000). Prenylation of the floral transcription tactor APETALA1 modulates its function. Plant Cell 12, 1257-1266. doi: 10.1105/tpc.12.8.1257

Yoo, S. K., Wu, X., Lee, J. S., and Ahn, J. H. (2011). AGAMOUS-LIKE 6 is a floral promoter that negatively regulates the FLC/MAF clade genes and positively regulates FT in Arabidopsis. Plant J. 65, 62-76. doi: 10.1111/j.1365313X.2010.04402.x

Yu, H., and Goh, C. J. (2000). Identification and characterization of three orchid MADS-box genes of the AP1/AGL9 subfamily during floral transition. Plant Physiol. 123, 1325-1336. doi: 10.1104/pp.123.4.1325

Zahn, L. M., Kong, H., Leebens-Mack, J. H., Kim, S., Soltis, P. S., Landherr, L. L., et al. (2005). The evolution of the SEPALLATA subfamily of MADS-Box genes: a preangiosperm origin with multiple duplications throughout angiosperm history. Genetics 169, 2209-2223. doi: 10.1534/genetics.104.037770

Conflict of Interest Statement: The authors declare that the research was conducted in the absence of any commercial or financial relationships that could be construed as a potential conflict of interest.

Copyright (C) 2015 McCarthy, Mohamed and Litt. This is an open-access article distributed under the terms of the Creative Commons Attribution License (CC BY). The use, distribution or reproduction in other forums is permitted, provided the original author(s) or licensor are credited and that the original publication in this journal is cited, in accordance with accepted academic practice. No use, distribution or reproduction is permitted which does not comply with these terms. 